\title{
Approximate Policy Iteration for Closed-Loop Learning of Visual Tasks
}

\author{
Sébastien Jodogne, Cyril Briquet, and Justus H. Piater \\ University of Liège - Montefiore Institute (B28) \\ B-4000 Liège, Belgium \\ \{S.Jodogne, C.Briquet, Justus.Piater\}@ULg.ac.be
}

\begin{abstract}
Approximate Policy Iteration (API) is a reinforcement learning paradigm that is able to solve high-dimensional, continuous control problems. We propose to exploit API for the closed-loop learning of mappings from images to actions. This approach requires a family of function approximators that maps visual percepts to a real-valued function. For this purpose, we use Regression Extra-Trees, a fast, yet accurate and versatile machine learning algorithm. The inputs of the Extra-Trees consist of a set of visual features that digest the informative patterns in the visual signal. We also show how to parallelize the Extra-Tree learning process to further reduce the computational expense, which is often essential in visual tasks. Experimental results on real-world images are given that indicate that the combination of API with Extra-Trees is a promising framework for the interactive learning of visual tasks.
\end{abstract}

\section{Introduction}

Since the rise of embedded CCD sensors, many robots are nowadays equipped with cameras and, as such, face input spaces that are extremely high-dimensional and potentially very noisy. Therefore, though real-world visual tasks can often be solved by directly connecting the visual space to the action space (i.e. by learning a direct image-to-action mapping), such mappings are especially hard to derive by hand and should be learned by the robotic agent. The latter class of problems is commonly referred to as Vision-for-Action. A breakthrough in modern AI would be to design an artificial system that would acquire object or scene recognition skills using only its interactions with the environment.

In this paper, an algorithm is introduced for closed-loop learning of imageto-action mappings. Our algorithm is defined within the biologically-inspired framework of reinforcement learning (RL) [1]. RL models an agent that learns a percept-to-action mapping through its interactions with the environment: It is only implicitly guided through a reinforcement signal, which is generally delayed.

$\mathrm{RL}$ is an attractive framework for Vision-for-Action problems. Unfortunately, basic RL algorithms are highly sensitive to the noise and to the dimensionality of the percepts, which forbids their direct use when solving visual tasks. We have previously proposed an algorithm that adaptively discretizes the visual space into a small number of visual classes [2]. Schematically, a decision tree is 
progressively built that tests, at each of its nodes, the presence of one highly informative image pattern (a visual feature). The tree is incrementally refined in a sequence of attempts to remove perceptual aliasing. This dramatically reduces the size of the input space, so that standard RL algorithms become usable.

One might wonder, however, whether the feature selection process is actually desirable, as it might introduce a high variance in the computed image classifiers, just as in the case of incremental learning of decision trees [3]. Furthermore, selecting visual features requires the introduction of an equivalence relation between the features. This relation is difficult to define. Often, a fixed threshold on a metric in feature space is used. However, modifying this threshold can lead to significant changes in the learned image-to-action mapping.

We describe a method that uses the whole set of visual features, without taking decisions involving a subset of informative features, and without relying on a similarity measure between them. In other words, we exploit the raw visual features. As visual data can be sampled only sparsely, we resort to the embedding of function approximators inside the RL process. Among the RL algorithms that use function approximators, we use Approximate Policy Iteration (API) [1]. API together with a linear approximation architecture has already been proposed in the context of continuous state spaces, giving rise to the Least-Squares Policy Iteration algorithm [4].

The Visual Approximate Policy Iteration (V-API) is defined, which is an instance of API designed to work in visual spaces. V-API uses Regression ExtraTrees, a family of nonparametric function approximators. This choice is motivated by the low bias and variance, as well as the good performance in generalization of the Extra-Trees [3. Furthermore, Classification Extra-Trees are successful for solving image classification tasks [5]. To the best of our knowledge, this makes of V-API the first application of API to high-dimensional discrete spaces. Thus, $\mathrm{V}$-API is potentially of major interest, as it proves that fully automatic, nonparametric RL methods can succeed in visual tasks. Likewise, the embedding of Extra-Trees in API is novel, and should also be useful in continuous state spaces.

Even if the computational expense of Extra-Trees is small with respect to other machine learning algorithms, the complexity of visual spaces still prevents their direct use in V-API. An additional contribution is to parallelize the ExtraTrees learning algorithm, which greatly reduces the execution time of V-API.

The paper is organized as follows. Firstly, we discuss the three important tools that are used inside V-API, namely the Modified Policy Iteration algorithm, the extraction of visual features and the Regression Extra-Trees. Then, we formally describe V-API and the distributed learning of Extra-Trees. Finally, we conclude with experimental results on a complex, visual navigation task.

\section{Theoretical Background}

\subsection{Modified Policy Iteration}

$\mathrm{V}$-API is defined in the framework of Reinforcement Learning (RL). In RL, the environment is traditionally modeled as a Markov Decision Process (MDP). An 
MDP is a quadruple $\langle S, A, \mathcal{T}, \mathcal{R}\rangle$, where $S$ is a finite set of states or percepts 1 , $A$ is a finite set of actions, $\mathcal{T}$ is a probabilistic transition function from $S \times$ $A$ to $S$, and $\mathcal{R}$ is a reinforcement signal from $S \times A$ to $\mathbb{R}$. An MDP obeys the following discrete-time dynamics: If at time $t$, the agent takes the action $a_{t}$ while the environment lies in a state $s_{t}$, the agent perceives a numerical reinforcement $r_{t+1}=\mathcal{R}\left(s_{t}, a_{t}\right)$, then reaches some state $s_{t+1}$ with probability $\mathcal{T}\left(s_{t}, a_{t}, s_{t+1}\right)$. Therefore, from the point of view of the agent, an interaction with the environment is summarized as a quadruple $\left\langle s_{t}, a_{t}, r_{t+1}, s_{t+1}\right\rangle$.

A (deterministic) percept-to-action mapping (or, equivalently, a control policy) is a fixed function $\pi: S \mapsto A$ from percepts to actions. Each control policy $\pi$ is associated with a state-action value function $Q^{\pi}(s, a)$ that gives, for each state $s \in S$ and each action $a \in A$, the expected discounted return obtained by starting from state $s$, taking action $a$, and thereafter following $\pi$ :

$$
Q^{\pi}(s, a)=\mathrm{E}^{\pi}\left\{\sum_{t=0}^{\infty} \gamma^{t} r_{t+1} \mid s_{0}=s, a_{0}=a\right\},
$$

where $\gamma \in[0,1[$ is the discount factor that gives the current value of the future reinforcements, and where $\mathrm{E}^{\pi}$ denotes the expected value if the agent follows the mapping $\pi$. Theory shows that all the optimal policies for a given MDP share the same $Q$ function, denoted $Q^{*}$ and called the optimal state-action value function, that always exists. Once the optimal state-action value function $Q^{*}$ is known, an optimal percept-to-action mapping $\pi^{*}$ is easily derived by choosing:

$$
\pi^{*}(s)=\underset{a \in A}{\operatorname{argmax}} Q^{*}(s, a), \text { for each } s \in S .
$$

Dynamic Programming (DP) is a set of algorithmic methods for solving MDPs. DP algorithms assume the knowledge of the transition function $\mathcal{T}$ and of the reinforcement signal $\mathcal{R}$. The well-known Modified Policy Iteration (MPI) [6] is an important DP algorithm that will be useful in the sequel. Starting with an initial, arbitrary percept-to-action mapping $\pi_{0}$, MPI builds a sequence of increasingly better policies $\pi_{1}, \pi_{2}, \ldots$ by relying on two interleaved learning processes: (1) policy estimation (the critic component), which computes the $Q^{\pi_{k}}$ state-action value function of the current policy $\pi_{k}$; and (2) policy improvement (the actor component), which uses $Q^{\pi_{k}}$ to generate an improved policy $\pi_{k+1}$. The algorithm stops when there is no change between successive policies. Here is a brief description of the two processes:

Policy estimation: $Q^{\pi_{k}}$ is computed by building a sequence of state-action value functions $Q_{0}, Q_{1}, \ldots$ until convergence using the update rule:

$$
Q_{i+1}(s, a)=\mathcal{R}(s, a)+\gamma \sum_{s^{\prime} \in S} \mathcal{T}\left(s, a, s^{\prime}\right) Q_{i}\left(s^{\prime}, \pi\left(s^{\prime}\right)\right) .
$$

After convergence, Bellman's theorem [1] shows that $Q_{i_{k}}=Q^{\pi_{k}} . Q_{0}$ can be chosen freely (generally, $Q_{0}(s, a)=0$ for each $s \in S$ and $a \in A$ ).

${ }^{1}$ MDP assumes the full observability of the environment, which allows us to talk indifferently about states and percepts. In visual tasks, $S$ is a set of images. 
Policy improvement: At each state $s \in S, \pi_{k}(s)$ is replaced by the action with the best state-action value (as computed by the policy estimation process):

$$
\pi_{k+1}(s)=\underset{a \in A}{\operatorname{argmax}} Q^{\pi_{k}}(s, a) .
$$

Finally, reinforcement learning is defined as the counterpart of DP when the transition function $\mathcal{T}$ and the reinforcement signal $\mathcal{R}$ are unknown. The input of RL algorithms is basically a database of interactions $\left\langle s_{t}, a_{t}, r_{t+1}, s_{t+1}\right\rangle$.

\subsection{Extraction of Visual Features}

Because standard RL algorithms rely on a tabular representation of the value functions, they quickly become impractical as the number of possible percepts increases. This is evidently a problem in visual tasks, as images are high-dimensional and noisy. Similar problems often arise in many fields of Computer Vision.

For this purpose, the popular, highly successful local-appearance methods have been introduced 78 . They postulate that, to take the right decision in a visual problem, it is often sufficient to focus one's attention only on a few interesting patterns occurring in the images. They summarize the images as a set of visual features, that are vectors of real numbers. Formally, they introduce a feature transform $F: S \mapsto \mathcal{P}\left(\mathbb{R}^{n}\right)$, where $S$ is the set of images and $\mathcal{P}$ denotes the power set. For an image $s \in S, F(s)$ typically contains between 10 and 1000 visual features. Most feature transforms have in common that (1) they select interest points in the image (e.g. by detecting discontinuities in the visual signal), and (2) they compute a local description of the neighborhood of the interest points. As an illustration, here are two possible choices of feature transforms:

1. "Traditional" feature transforms, that use a standard interest point detector (Harris, Harris-affine, Hessian-Laplace,...) [7] in conjunction with a standard local descriptor (steerable filters, local jet, SIFT,... [ 8].

2. Randomized feature transforms. They randomly select a fixed number of subwindows in the image (a subwindow is a rectangle that has an arbitrary position, scale and orientation). Then, the subwindows are downscaled to a patch of fixed size (typically $11 \times 11$ ), in a fixed colorspace (graylevel, RGB or HSV). This simple approach is very fast, as well as highly successful [5].

V-API uses such methods to digest an image into a set of raw visual features.

\subsection{Regression Extra-Trees}

As discussed in the Introduction, V-API rely on Extra-Trees [3. We restrict our study of Extra-Trees to the case where all attributes (both the inputs and the output) are numerical. An Extra-Tree model is constituted by a forest of $M$ independent decision trees. Each of their internal nodes is labeled by a threshold on one of the input attributes, that is to be tested in that node. The leaves are labeled by a regression output. The regression response for a sample is obtained by computing the response of each subtree. This is achieved by starting 
at the root node, then progressing down the tree according to the result of the thresholding tests found during the descent, until a leaf is reached. By doing so, each subtree votes for a regression output. Finally, the mean of these outputs is assigned to the sample.

The subtrees are built in a top-down fashion, by successively splitting the leaf nodes where the output variable varies. For each input variable, the algorithm computes its variation bounds and uniformly chooses one random threshold between those bounds. Once a threshold has been chosen for every input variable, the split that gives the best score on the regression output is kept. The tree construction is stopped when the output is constant in the leaf. This will guarantee that learning bias is small, as well as learning variance thanks to the aggregation of a sufficient number of randomized trees.

Algorithms 1 and 2 describe how to build an Extra-Tree model. In this pseudocode, $\boldsymbol{x}_{i} \in \mathbb{R}^{n}$ contains the input attributes of the $i$ th sample in the learning set and $y_{i} \in \mathbb{R}$ is the observed regression output for this sample. We assume the existence of a function $\operatorname{score}\left(\left\{\left\langle\boldsymbol{x}_{i}, y_{i}\right\rangle\right\}, v, t\right)$ that returns the score of the threshold $t$ on the variable $v$ in the database $\left\{\left\langle\boldsymbol{x}_{i}, y_{i}\right\rangle\right\}$. In our implementation, variance reduction was used as the score function. These algorithms are identical to those presented by Geurts et al. 3. and are restated here to complement the description of our distributed algorithms (cf. Section [3.5). We refer the reader to Geurts et al. 3] for a complete and thorough treatment.

\section{Visual Approximate Policy Iteration}

\subsection{Nonparametric Approximate Policy Iteration}

In the next sections, a general, nonparametric RL version of Approximate Policy Iteration (API) is described. Nonparametric API is a generalization of MPI that can use any kind of nonparametric function approximators. This is in contrast to Least-Squares Policy Iteration [4] that explicitly targets continuous state spaces and uses linear approximation. The two components of MPI (policy estimation and improvement) are adapted so as to compute the state-action value function of a policy without relying on any knowledge of the underlying MDP.

The existence of an oracle called learn is assumed, as we are concerned with nonparametric function approximators. Given a database of samples $\left\langle s_{t}, a_{t}, v_{t}\right\rangle$, where $s_{t}$ is a state, $a_{t}$ is an action and $v_{t}$ is a real number, learn builds a function approximator that represents a state-action value function $Q: S \times A \mapsto \mathbb{R}$ that is the closest possible to the given sample distribution. For instance, Algorithm 1 constitutes one possible oracle that is suitable for problems with continuous state space. An oracle for visual spaces will be introduced in Section 3.4.

\subsection{Representation of the Generated Policies}

Nonparametric API relies on the state-of-the-art principle that was proposed in Least-Squares Policy Iteration [4: Any state-action value function $Q(s, a)$ induces a greedy policy $\widetilde{\pi}[Q]$ that always selects the action maximizing $Q$ : 

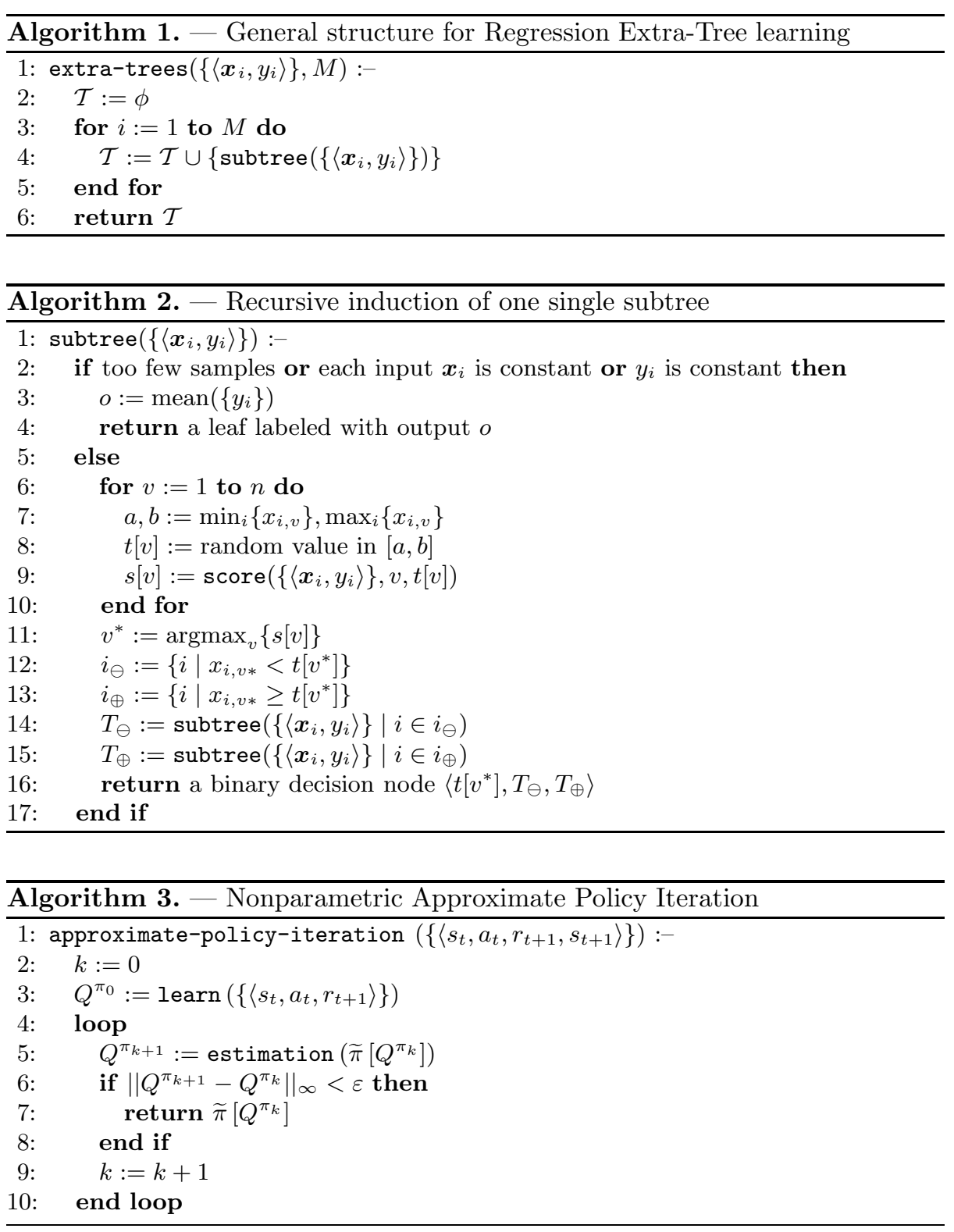

$$
\widetilde{\pi}[Q](s)=\underset{a \in A}{\operatorname{argmax}} Q(s, a), \text { for each } s \in S
$$

This property enables us to deal only with state-action value functions, and never directly with policies. So, there is no need of a separate representation system for policies. In terms of the notation of Section 2.1 nonparametric API

\footnotetext{
${ }^{2}$ This maximization can easily be achieved, as the action space is assumed to be finite.
} 


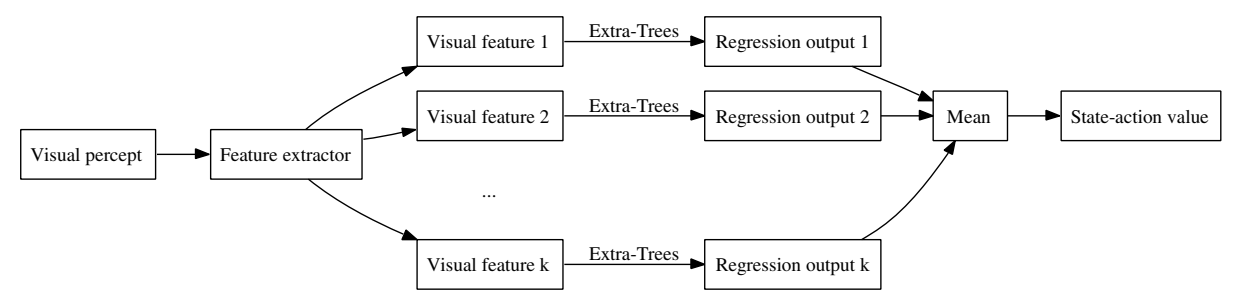

Fig. 1. Computing the state-action value of a visual percept for a given action

does not keep track of $\pi_{k}=\widetilde{\pi}\left[Q^{\pi_{k-1}}\right]$, but only of $Q^{\pi_{k}}$. The policy $\pi_{k}$ is evaluated on demand from $Q^{\pi_{k-1}}$ through Equation 5 . Thanks to this implicit representation of the policies, the policy improvement step becomes trivial: It is sufficient to define $Q^{\pi_{k+1}}$ as the state-action value function of the greedy policy with respect to $Q^{\pi_{k}}$, that is computed by the policy estimation component.

Algorithm 3 summarizes the backbone of nonparametric API. The input of the algorithm is a database of interactions $\left\langle s_{t}, a_{t}, r_{t+1}, s_{t+1}\right\rangle$, which makes of nonparametric API an off-policy, model-free RL algorithm. The third line builds an initial policy $\pi_{0}$ that maximizes immediate reinforcements. The algorithm stops when the difference between two successive $Q^{\pi_{k}}$ drops below a threshold.

\subsection{Nonparametric Approximate Policy Estimation}

The estimation component in Algorithm 3 computes the state-action value function $Q^{\pi_{k+1}}$ of a policy $\widetilde{\pi}\left[Q^{\pi_{k}}\right]$ given the input database of interactions $\left\langle s_{t}, a_{t}, r_{t+1}, s_{t+1}\right\rangle$. To this end, a sequence of state-action value functions $Q_{i}(s, a)$ is generated. This is done according to the principle of Modified Policy Iteration, but the functions $Q_{i}$ are now function approximators that are built through the learn oracle. As the underlying MDP is unknown, the update rule of Equation 3 cannot be used. Instead, we use the stochastic version of this equation:

$$
Q_{i+1}(s, a)=\mathcal{R}(s, a)+\gamma Q_{i}\left(\delta(s, a), \widetilde{\pi}\left[Q^{\pi_{k}}\right](\delta(s, a))\right) .
$$

Hence, the update rule that is induced by the database of interactions is:

$$
Q_{i+1}:=\operatorname{learn}\left(\left\{\left\langle s_{t}, a_{t}, r_{t+1}+\gamma Q_{i}\left(s_{t+1}, \widetilde{\pi}\left[Q^{\pi_{k}}\right]\left(s_{t+1}\right)\right)\right\rangle\right\}\right) .
$$

A new Extra-tree model is learned through each application of this update rule. This learning process stops when the difference between two successive $Q_{i}$ drops below a threshold. $Q_{0}$ can be chosen freely. In practice, $Q_{0}$ is set to $Q^{\pi_{k}}$. This is a starting point that reduces the number of iterations before convergence, as the policy $\widetilde{\pi}\left[Q^{\pi_{k+1}}\right]$ generally shares common decisions with $\widetilde{\pi}\left[Q^{\pi_{k}}\right]$. This algorithm can be motivated similarly than Fitted $Q$ Iteration [9]: The stochastic aspect of the environment will eventually be captured by the function approximators.

\subsection{Visual State-Action Value Function Approximators}

So far, we have not defined a family of function approximators that is suitable for visual tasks. As motivated in the Introduction, we propose to take advantage 
of the raw visual features. Therefore, Regression Extra-Trees cannot be used directly, for two reasons: (1) The action input is discrete, and cannot be fed into a Regression Extra-Tree model as defined in Section 2.3, and (2) feature transforms map one visual percept to many visual features (cf. Section 2.2).

The solution to the first problem is straightforward: The single Extra-Tree model $Q(s, a)$ is replaced by $|A|$ Extra-Tree models $Q_{a}(s)$, one for each possible action $a \in A$. The latter problem is more fundamental, and is solved by applying the Extra-Trees model independently on each visual feature in the input image. This process generates one regression output per visual feature. Then the value of the function approximator is defined as the mean of these regression outputs. This approach is depicted in Figure 1, and is directly inspired by recent, successful results about image classification through Extra-Trees [5].

The learn oracle for this type of function approximators is now defined formally. Given a database of samples $\left\langle s_{t}, a_{t}, v_{t}\right\rangle$ (where $s_{t}$ are images), the ExtraTrees model $Q_{a}$ that corresponds to the action $a \in A$ is defined as:

$$
Q_{a}(s):=\text { extra-trees }\left(\left\{\left\langle\boldsymbol{x}_{i}, y_{i}\right\rangle \mid\left(\exists t \in T_{a}\right)\left(\boldsymbol{x}_{i} \in F\left(s_{t}\right) \wedge y_{i}=v_{t}\right)\right\} ; M\right),
$$

where $F$ is the used feature transform, and $T_{a}=\left\{t \mid a_{t}=a\right\}$ is the set of time stamps for the interactions that are labeled with the action $a$. Intuitively, for each sample $\left\langle s_{t}, a_{t}, v_{t}\right\rangle$, all the visual features in the image $s_{t}$ are associated with the value $v_{t}$ in the database that is used to train the Extra-Tree model $Q_{a_{t}}$.

Nonparametric API along with such a family of function approximators will be referred to as the Visual Approximate Policy Iteration (V-API) algorithm.

\subsection{Parallelizing Extra-Trees}

V-API applies Regression Extra-Trees on large databases. This induces a high computational cost for the learning of Extra-Trees. We propose to reduce this computational expense by taking advantage of the extremely parallelizable nature of Algorithm 1] Each execution of Algorithm 2 is totally independent of other instances of the same algorithm, and each subtree can be computed in a separate computational task. In other words, the learning of Extra-Tree models can be formulated as a so-called bag of tasks, where tasks are independent and can be processed on separate computer nodes. Once all the tasks are completed, it is straightforward to merge all the subtrees to generate the Extra-Tree model.

Our implementation of Regression Extra-Trees follows this principle. The resulting speedup is huge: If $N$ homogeneous hosts are used, the computation time roughly equals $\lceil M / N\rceil T+U$, where $M$ is the parameter of Algorithm 1, $T$ is the mean amount of time for building one single subtree, and $U$ corresponds to the distribution time of the database among all the hosts.

Note that if no attention is paid, the transmission overhead $U$ can quickly become a bottleneck. Indeed, the same large database has to be sent to $N$ hosts by the central task manager, which causes both reduction of bandwidth and augmentation of network congestion effects. One possible solution is to rely on the use of UDP multicast. Unfortunately, due to the lack of flow control in UDP, slow hosts will be overwhelmed by the massive amount of data they receive. 
We have therefore used the peer-to-peer BitTorrent protocol [10] to distribute the databases. Schematically, in BitTorrent, each host becomes part of a swarm that grabs all the pieces of a file. Whenever a host acquires a piece, this piece is made available for download to the other hosts. A distinguished host, the tracker, is used to keep track of the hosts that belong to the swarm. This approach for file distribution is elegant and scalable, and indeed highly reduces the transmission overhead $U$, making it roughly independent of the number of hosts in the cluster. Our solution should be useful in many other distributed computing applications, and especially in the context of distributed data mining and Grid computing.

\section{Experimental Results}

We have applied the V-API to a simulated navigation task. In this task, the agent moves between 11 distinct locations of our campus (cf. Figure2(a)). Every time the agent is at one of the 11 locations, its body can aim at 4 possible orientations: North, South, West, East. It can take 3 different actions: Turn left, turn right, go forward. Its goal is to enter the Montefiore Institute, where it gets a reward of 100. Turning left or right induces a penalty of -5 , and moving forward, a penalty of -10 . The discount factor $\gamma$ was set to 0.8 .

The agent does not have access to its position and its orientation. Rather, it only perceives a picture of the area that is in front of it. So, the agent has to connect images directly to the appropriate reactions without knowing the underlying physical structure of the task. For each possible location and each possible viewing direction, a database of 24 images of size $1024 \times 768$ with viewpoint changes was collected. Those 44 databases were divided into a learning set of 18 images and a test set of 6 images (cf. Figure 2 (b)). V-API has been applied on a static database of 10,000 interactions that has been collected using a fully randomized exploration policy. The same database is used throughout the entire $\mathrm{V}$-API algorithm, and only contains images that belong to the learning set.

(a)

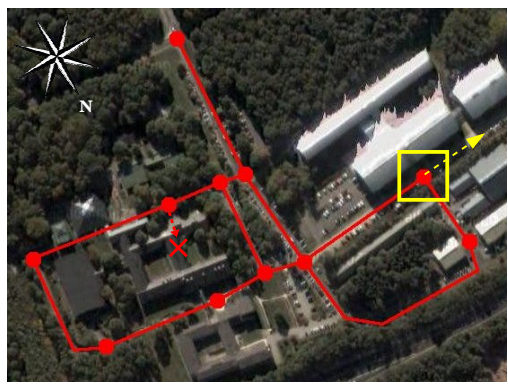

(b)

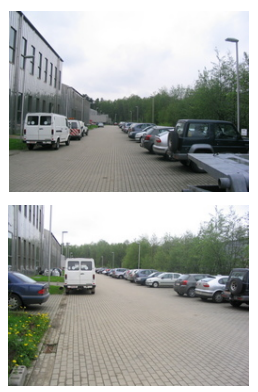

Fig. 2. (a) Navigation around Montefiore Institute. Red spots corresponds to the places between which the agent moves. The agent can only follow the links between the different spots. On this map, Montefiore Institute is labeled by a red cross. (b) The percepts of the agent. Four different percepts are shown that correspond to the location and viewing direction marked in yellow on the map on the left. 

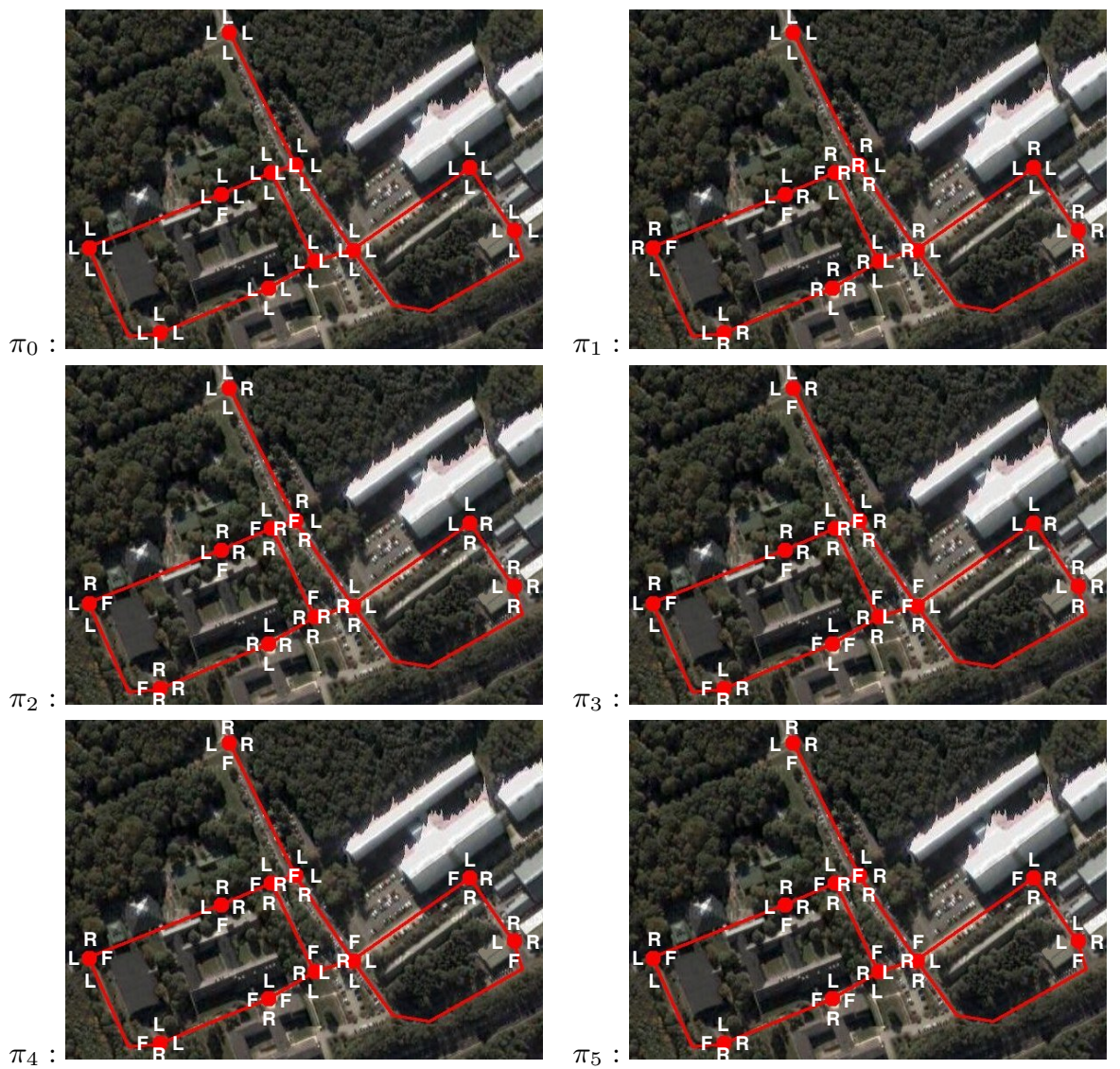

Fig. 3. The sequence of policies $\pi_{k}$ generated by V-API. At each location, 4 letters from the set $\{F, L, R\}$ are written, one for each viewing direction. Each letter represents the action (go Forward, turn Left, turn Right) that receives the majority of votes in the learning set, for the corresponding pair location / viewing direction.

In our experiments, we have used traditional feature transforms with the SIFT descriptors (whose dimension is $n=128$ ) [11. This choice is mostly arbitrary. Randomized feature transforms are promising, and will be investigated in future work. The parallel implementation of Regression Extra-Trees runs on a testbed cluster of $N=67$ heterogeneous ix86 machines. It consists of a set of $27 \mathrm{AMD}$ Athlon 1800+, 27 Intel Celeron 2.4Ghz, and 13 Intel Pentium IV 2.8Ghz CPUs. They are interconnected via a switched 100Mbps Ethernet network.

Figure 3 shows the sequence of policies that is generated by V-API. The algorithm stops after 6 iterations, which is a surprisingly small number. A total of 147 Extra-Tree models were generated that correspond to $49=147 /|A|$ visual state-action value functions (as defined in Section 3.4). The overall running time was about 98 hours. This shows the interest of taking advantage of the intrinsic parallelism of Extra-Trees, as this has divided the running time by about fifty. 


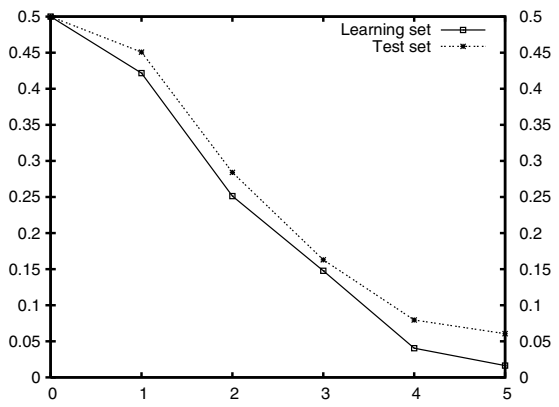

Fig. 4. Policy error as a function of the step counter $k$. The solid (resp. dashed) plot corresponds to the error rate of the policy $\pi_{k}$ on the learning (resp. test) set.

Statistics about the generated policies are given in Figure 4 . The error of the last policy in the generated sequence was $1.6 \%$ on the learning set and $6.6 \%$ on the test set, with respect to the optimal policy when the agent has direct access to its position and viewing direction. These error rates are better than those obtained through RLVC (2\% on the learning and $14 \%$ on the test set) [12].

The advantage of using BitTorrent is clear: In optimal conditions, if $m$ ExtraTree models (as discussed above, $m=147$ ) are to be built from databases of typical size $S=80 \mathrm{MB}$, the transmission overhead corresponding to FTP would roughly equal $U \approx m \times N \times S[\mathrm{MB}] / 100[\mathrm{Mbps}]=17$ hours. Using BitTorrent, this time is approximately reduced to $U \approx m \times S[\mathrm{MB}] / 100[\mathrm{Mbps}]=16$ minutes.

\section{Conclusions}

We have introduced the Visual Approximate Policy Iteration (V-API) algorithm. $\mathrm{V}$-API is designed for the closed-loop solution of visual control problems. It extensively relies on the use of Regression Extra-Trees as function approximators. The embedding of Extra-Trees inside the framework of API is a first important contribution of this paper. Experiments indicate that the algorithm is sound and applicable to non-trivial visual tasks. V-API outperforms RLVC in terms of performance in generalization over the test set. Future work will demonstrate that the proposed combination of nonparametric API with Extra-Trees is a convenient choice for RL in high-dimensional, continuous state spaces.

We have also shown how to take advantage of the highly parallelizable nature of the induction of Extra-Trees by distributing the construction of the subtrees over a cluster of computers. This allows us to greatly reduce the computational time, which is often an important issue with visual spaces. Of course, other fields of application of Extra-Trees, such as supervised learning [3], image classification [5] and the Fitted $Q$ Iteration algorithm [9], will directly benefit from this new economical advantage. Finally, the peer-to-peer BitTorrent protocol was shown to be an effective tool for reducing the database distribution expense.

Unfortunately, even when taking advantage of a cluster of computers, the running time of the algorithm is still relatively long. A compromise seems to 
exist between the requirement of an equivalence relation among visual features, as in the algorithm RLVC [2], and the use of raw visual features, as in V-API. RLVC runs faster, does not require a cluster of computers, and can be used to generate higher-level visual features [13. On the other hand, V-API benefits from the full discriminative power of the visual features, exhibits the low variance and bias of Extra-Trees, and requires less parameter tuning. Therefore, V-API and RLVC constitute two complementary techniques. An interesting open question is whether the advantages of these two techniques can be combined.

Acknowledgments. We kindly acknowledge P. GeurTs and the PePITe s.A. team (http://www.pepite.be/) for providing us with an implementation of the Extra-Trees, which has inspired our parallel implementation. We also wish to thank Prof. L. WEHENKEL for helpful comments on a preliminary version of this paper, and S. MARTIN for his valuable help regarding network programming and BitTorrent's protocol.

\section{References}

1. Bertsekas, D., Tsitsiklis, J.: Neuro-Dynamic Programming. Athena Scientific (1996)

2. Jodogne, S., Piater, J.: Interactive learning of mappings from visual percepts to actions. In De Raedt, L., Wrobel, S., eds.: Proc. of the 22nd International Conference on Machine Learning (ICML), Bonn (Germany), ACM (2005) 393-400

3. Geurts, P., Ernst, D., Wehenkel, L.: Extremely randomized trees. Machine Learning 36(1) (2006) 3-42

4. Lagoudakis, M., Parr, R.: Least-squares policy iteration. Journal of Machine Learning Research 4 (2003) 1107-1149

5. Marée, R., Geurts, P., Piater, J., Wehenkel, L.: Random subwindows for robust image classification. In: IEEE Conference on Computer Vision and Pattern Recognition. Volume 1., San Diego (CA, USA) (2005) 34-40

6. Puterman, M., Shin, M.: Modified policy iteration algorithms for discounted Markov decision problems. Management Science 24 (1978) 1127-1137

7. Schmid, C., Mohr, R., Bauckhage, C.: Evaluation of interest point detectors. International Journal of Computer Vision 37(2) (2000) 151-172

8. Mikolajczyk, K., Schmid, C.: A performance evaluation of local descriptors. In: Proc. of the IEEE Conference on Computer Vision and Pattern Recognition. Volume 2., Madison (WI, USA) (2003) 257-263

9. Ernst, D., Geurts, P., Wehenkel, L.: Tree-based batch mode reinforcement learning. Journal of Machine Learning Research 6 (2005) 503-556

10. Cohen, B.: Incentives build robustness in BitTorrent. In: Proc. of the Workshop on Economics of Peer-to-Peer Systems. (2003)

11. Lowe, D.: Distinctive image features from scale-invariant keypoints. International Journal of Computer Vision 60(2) (2004) 91-110

12. Jodogne, S., Piater, J.: Learning, then compacting visual policies (extended abstract). In: Proc. of the 7th European Workshop on Reinforcement Learning (EWRL), Napoli (Italy)(2005) 8-10

13. Jodogne, S., Scalzo, F., Piater, J.: Task-driven learning of spatial combinations of visual features. In: Proc. of the IEEE Workshop on Learning in Computer Vision and Pattern Recognition, San Diego (CA, USA), IEEE (2005) 